\title{
CONSUMO DE ESTEROIDES ANABOLIZANTES POR PRATICANTES DE EXERCÍCIOS FÍSICOS EM ACADEMIAS DE PRESIDENTE PRUDENTE
}

Rafael Dias Montanher, Robson Chacon Castoldi, Carlos Augusto de Carvalho Filho, Ariovaldo de Souza Ribeiro, Adriana Junqueira

Universidade do Oeste Paulista - UNOESTE, Curso de Educação Física, Presidente Prudente, SP. E-mail: rafadmontanher@gmail.com.

\section{RESUMO}

Usados indiscriminadamente, os esteroides androgênicos anabolizantes (EAAs), suplementos alimentares (SAs) e termogênicos estão entre os agentes ergogênicos mais procurados para obter um estereótipo de corpo perfeito. O presente estudo teve como objetivo analisar a prevalência do uso de EAAs entre os praticantes de atividade física de academias de Presidente Prudente. Foi criado um questionário semiestruturado, autoaplicável e anônimo para obtenção dos dados. Foram respondidos 120 questionários no intervalo de uma semana, e as respostas caracterizaram que $76,7 \%$ dos entrevistados estava fazendo uso de alguma substância ergogênica, 10\% relatou uso de esteroides anabolizantes, $22,5 \%$ termogênicos e $44,2 \%$ suplementos alimentares, com prevalência para o sexo masculino. Esses dados demonstram que os EAA são uma realidade nas academias da cidade de Presidente Prudente/SP. São usados entre pessoas com maior grau de instrução, jovens e do sexo masculino.

Palavras Chaves: recursos ergogênicos, atletas, treinamento de resistência, estética, doping.

\section{CONSUMPTION OF ANABOLIC STEROIDS BY PHYSICAL EXERCISE PRACTITIONERS IN PRUDENT PRESIDENT'S ACADEMIES.}

\begin{abstract}
Used indiscriminately, anabolic androgenic steroids (AAS), dietary supplements (SAs) and thermogenics are among the most sought-after ergogenic agents to achieve a perfect body stereotype. The present study aimed to analyze the prevalence of AAS use among physical activity practitioners at academies of the Presidente Prudente. A semi-structured, self-administered and anonymous questionnaire was created to obtain the data. A total of 120 questionnaires were answered within one week, and responses $76.7 \%$ of respondents are using some ergogenic substance, $10 \%$ reported use of anabolic steroids, $22.5 \%$ thermogenic and $44.2 \%$ dietary supplements, with prevalence for males. These data demonstrate that AAS are a reality in the academies of the city of Presidente Prudente/SP. They are used among people with higher education, young and male.
\end{abstract}

Keywords: ergogenic resources, athletes, resistance training, esthetics, doping.

\section{INTRODUÇÃO}

A busca do "corpo perfeito" tem sido cada vez mais almejada, no intuito de se obter um maior reconhecimento pessoal e profissional $^{1}$. Entre os meios utilizados para se atingir um ideal estético destaca-se o uso de fármacos para emagrecer, uso de esteróides anabólicos para os músculos, e cirurgias plásticas. Os anabolizantes estão entre os fármacos procurados para atingir este ideal ${ }^{2,3}$. O uso indiscriminado desses medicamentos e de moderadores de apetite tem aumentado muito entre os praticantes de exercícios físicos ${ }^{4}$.

Os esteróides androgênicos anabólicos (EAAs) são substâncias químicas derivadas da testosterona, hormônio masculino, que é uma substância fisiológica produzida pelo organismo e sintetizada pela indústria farmacêutica a fim de 
solucionar patologias, mas, são usadas indevidamente por atletas e praticantes de exercícios físicos visando principalmente à melhora do rendimento, aumento da força e massa muscular, pois são altamente sintetizados pelo nosso organismo, aumentando a síntese proteica no músculo ${ }^{5}$.

É conhecida a prescrição dos anabolizantes para diversos tipos de tratamentos de doenças crônicas associadas a um catabolismo acentuado, tais como: condições de AIDS, doenças pulmonares obstrutivas crônicas, deficiência hepática ou renal, câncer, casos de queimaduras e recuperação pós-cirurgia ${ }^{6,7}$. No entanto, o uso desses anabolizantes sem prescrições médicas tem despertado a atenção da comunidade científica pelo seu uso abusivo, tornando-se um problema de saúde pública, pois, a população jovem e adulta nem sempre conhece os efeitos adversos causados pelo uso indiscriminado dessas substâncias ${ }^{8,9}$.

São vários os efeitos colaterais causados pelo uso indiscriminado de EAAs, desde uma simples cãibra e cansaço muscular, até prejuízos graves, como a acromegalia, síndrome metabólica, sobrecargas renais e hepáticas, alterações psicológicas, cardíacas e morfológicas (ginecomastia e aumento do clitóris), como também situações fatais ${ }^{10,11}$.

Atletas que utilizam EAAs podem sofrer alguma mudança de comportamento, que evolui desde uma simples alteração de humor a uma psicose que requer tratamento e hospitalização ${ }^{12}$. Há um fator psicológico que faz com que os usuários de EAAs tenham uma sensação aumentada de bem estar e de autoconfiança, além da exacerbação de comportamento agressivo $^{13}$.

No Brasil, alguns trabalhos de caráter epidemiológico foram realizados com o intuito de apresentar dados regionais sobre a incidência do uso de EAAs na população ${ }^{14}$. Diante do aumento da oferta e da procura de diversos recursos ergogênicos, entre eles os EAAs, além da ausência de estudos com caráter epidemiológico na região Oeste do Estado de São Paulo, este estudo teve o objetivo de avaliar indivíduos praticantes de exercícios físicos em academias de Presidente Prudente, e a relação com suas atitudes e práticas em saúde, é uma importante estratégia para sinalizar sua prevalência. Além disso, procurou-se caracterizar o perfil desses usuários no que diz respeito ao grau de instrução, faixa etária e gênero.

\section{METODOLOGIA}

Este estudo caracterizou-se como investigativo, descritivo, com delineamento transversal, na forma de questionário, com metodologia quantitativa, foi submetido à avaliação e apreciação do Comitê de Ética em Pesquisa (CEP) da Universidade do Oeste Paulista (UNOESTE) e aprovada sob o número CAAE 64995817.4.0000.5515.

Participaram desta pesquisa 120 praticantes de atividade física de três grandes academias da cidade de Presidente Prudente, cadastradas no Conselho Regional de Educação Física 4a Região (CREF4), que constituíram a população alvo deste estudo.

Foi elaborado um questionário semiestruturado, autoaplicável, para obter dados referentes ao gênero, à idade, grau de instrução e ao uso de suplementos alimentares, termogênicos e esteroides anabolizantes (EAA), em formato de fácil resposta sim/não e de múltipla escolha, respondidos de forma anônima e voluntaria pelos praticantes.

Os questionários ficaram disponíveis nas academias, durante uma semana, onde os participantes foram orientados e incentivados a participarem do estudo respondendo o questionário. Todos os responsáveis pelas academias assinaram um termo de responsabilidade e compromisso, assegurando o anonimato dos participantes e dos estabelecimentos, como também da confidencialidade dos resultados para a realização desta pesquisa. Após o preenchimento, os questionários foram depositados pelos próprios voluntários em urnas lacradas.

Fizeram parte do estudo alunos e professores de educação física de ambos os sexos, maiores de 18 anos, atuantes em academias de Presidente Prudente/SP. Foram excluídos questionários com respostas incompletas e pessoas incapazes de compreender e responder o questionário.

A análise dos resultados foi realizada e apresentada a partir da frequência das respostas obtidas. Todos os dados foram organizados e desenvolvidos utilizando o programa Microsoft 
Office Excel 2010 para análise descritiva e construção dos gráficos.

\section{RESULTADOS}

Este estudo realizado em Presidente Prudente/SP obteve, do total de 200 questionários distribuídos, 120 (60\%) questionários respondidos.

Os participantes foram de ambos os sexos, sendo $41 \%$ do sexo feminino e $59 \%$ do sexo masculino.

Dos 120 entrevistados $10 \%$ declararam utilizarem EAAs, 22,5\% utilizam substâncias termogênicos, $44,2 \%$ utilizam SAs e $23,3 \%$ declararam nunca terem utilizado nenhuma destas substâncias. De acordo com o levantamento dos questionários, $76,7 \%$ dos entrevistados que utiliza alguma destas substâncias ergogênicas, prevalecem pessoas com faixa etária entre 18 a 23 anos (39,2\%), com nível de escolaridade em ensino superior $(47,5 \%)$ e do gênero masculino $(45,8 \%)$ (Tabela 1$)$.

Outro aspecto analisado entre os praticantes de atividade física e usuários de EAA foi sobre o conhecimento dos efeitos indesejáveis dos anabolizantes e seus malefícios, a maioria $(82,5 \%)$ declarou total desconhecimento (Figura 1).

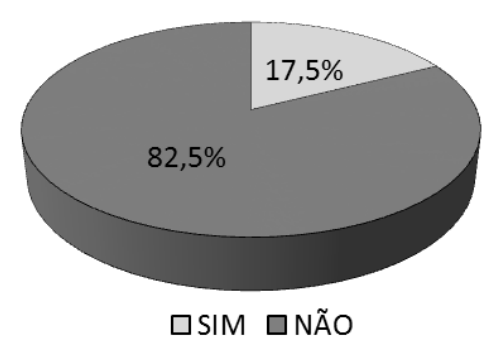

Figura 1. Ciência sobre os efeitos indesejáveis dos anabolizantes em participantes de ambos os sexos.

Quando interrogados sobre a orientação profissional, apenas $17,5 \%$ dos entrevistados declararam fazer uso de EAAs com orientação (Figura 2).

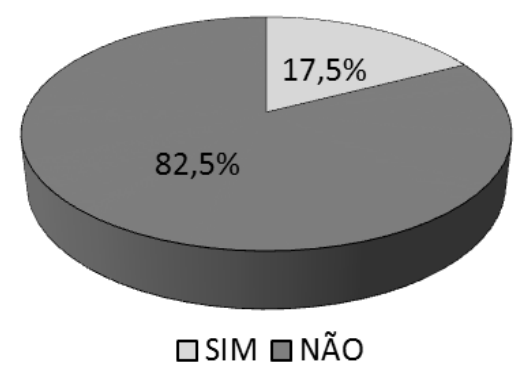

Figura 2. Uso de SAs, Termogênicos e EAA com orientação profissional.

Tabela 1. Perfil dos consumidores de Suplementos Alimentares (SAs), Termogênicos e Esteroides Androgênicos Anabolizantes (EAA).

\begin{tabular}{|c|c|c|c|c|c|c|c|c|}
\hline \multirow[t]{2}{*}{ Variáveis } & \multicolumn{2}{|c|}{ SAs } & \multicolumn{2}{|c|}{ Termogênicos } & \multicolumn{2}{|c|}{ EAA } & \multicolumn{2}{|c|}{ Total uso } \\
\hline & $\mathrm{N}$ & $\%$ & $\mathrm{~N}$ & $\%$ & $\mathrm{~N}$ & $\%$ & $\mathrm{~N}$ & $\%$ \\
\hline Não usuário & 67 & 55,8 & 93 & 77,5 & 108 & 90 & 28 & 23,3 \\
\hline \multicolumn{9}{|l|}{ Faixa etária } \\
\hline 18 a 23 anos & 21 & 17,5 & 16 & 13,3 & 10 & 8,3 & 47 & 39,1 \\
\hline 24 a 29 anos & 16 & 13,3 & 6 & 5,0 & 2 & 1,7 & 24 & 20,0 \\
\hline 30 a 35 anos & 11 & 9,2 & 3 & 2,5 & 0 & 0 & 14 & 11,7 \\
\hline$>$ de 36 anos & 5 & 4,2 & 2 & 1,7 & 0 & 0 & 7 & 5,9 \\
\hline Total & 120 & 100 & 120 & 100 & 120 & 100 & 120 & 100 \\
\hline \multicolumn{9}{|l|}{ Escolaridade } \\
\hline Ensino Fundamental & 0 & 0 & 0 & 0 & 0 & 0 & 0 & 0 \\
\hline Ensino Médio & 20 & 16,4 & 9 & 7,5 & 6 & 5 & 35 & 29,2 \\
\hline Ensino Superior & 33 & 27,5 & 18 & 15 & 6 & 5 & 57 & 47,5 \\
\hline \multicolumn{9}{|l|}{ Gênero } \\
\hline Masculino & 37 & 30,8 & 9 & 7,5 & 9 & 7,5 & 55 & 45,8 \\
\hline Feminino & 16 & 13,3 & 18 & 15,0 & 3 & 2,5 & 37 & 30,8 \\
\hline
\end{tabular}




\section{DISCUSSÃO}

O presente estudo objetivou realizar, entre os praticantes de atividade física das academias de Presidente Prudente, uma análise epidemiológica do uso de recursos ergogênicos, entre eles os EAA, para sinalizar sua prevalência na região Oeste do Estado de São Paulo, além de caracterizar o perfil desses usuários em relação ao grau de instrução, faixa etária e gênero.

Em estudo realizado na cidade de Betim/MG, Ferreira et al. ${ }^{15}$ relataram que $62 \%$ dos participantes faz ou já fizeram uso de EAA. Em contrapartida, em nosso estudo, na cidade de Presidente Prudente/SP OS usuários representaram a minoria. O que corrobora com outros achados literários, Silva e Moreau ${ }^{16}$, declararam que $8 \%$ dos entrevistados estavam utilizando EAA, pesquisa realizada na cidade de São Paulo/SP e $10 \%$ no estudo de Carneiro-Junior et al. ${ }^{17}$ que pesquisou a cidade de Ubá/MG. O desconhecimento dos efeitos indesejáveis dos anabolizantes e seus malefícios, representaram a maioria dos participantes neste estudo, assim como no estudo de Mineiro et al. ${ }^{18}$, reportando que a desinformação gera maus hábitos que podem resultar em diversos danos à saúde.

Quanto ao grau de escolaridade, maior parte da amostra possui o ensino superior, seguido do ensino médio (Tabela 1), dados similares a estes foram encontrados por Frizon et al. ${ }^{19}$ onde os frequentadores de academias pesquisados dos municípios de Erechim e Passo Fundo-RS, 62,7\% relataram cursar ou ter concluído o nível superior e 33,7\% o Ensino Médio, enquanto Mineiro et al. ${ }^{18}$, Iriart ${ }^{11}$ e Santos et al. ${ }^{20}$, relataram que o ensino médio prevaleceu sobre o ensino superior para o uso de EAA, demonstrando a prevalência do uso de recursos ergogênicos entre pessoas com maior grau de instrução. Determinando assim, a necessidade da implantação de programas de conscientização durante o ensino médio, que é a base para o ensino superior. Entretanto, faz-se necessário um número maior de pesquisas para o enriquecimento das discussões sobre o assunto.

Em resposta aos objetivos propostos neste trabalho podemos constatar que a maioria dos usuários de EAA se encontra entre indivíduos do sexo masculino, na faixa etária de 18 a 29 anos, estando assim de acordo com diversos achados na literatura que aponta similaridade de gênero e faixa etária ${ }^{16-19,21}$.

\section{CONCLUSÃO}

Foi possível concluir que os EAA são uma realidade nas academias da cidade de Presidente Prudente/SP. São usados entre pessoas com maior grau de instrução, jovens e do sexo masculino que buscam acelerar os resultados esperados pelo treino, geralmente voltados à estética. A maioria dos usuários não sabe sobre os malefícios destas drogas e raramente têm acompanhamento médico especializado.

\section{CONFLITO DE INTERESSE}

Os autores declaram não haver qualquer potencial conflito de interesse que possa interferir na imparcialidade deste trabalho científico.

\section{REFERÊNCIAS}

1. Swami V, Frederick DA, Aavik T, Alcalay L, Allik J, Anderson $D$ et al. The attractive female body weight and female body dissatisfaction in 26 countries across 10 world regions: results of the international body project I. Pers Soc Psychol Bull. 2010;36(3):309-25. DOI: https://doi.org/10.1177/0146167209359702

2. Moriconi LF, da Silva TLC, Cardoso MR. Patologias alimentares e adolescência: a questão do feminino; adolescentes. São Paulo: Escuta; 2006.

3. Iriart JA, Andrade TM. Musculação o uso de esteroides anabolizantes e percepção de risco entre jovens fisiculturistas de um bairro popular de Salvador, Bahia, Brasil. Cad Saúde Pública. 2002;18(5):1379-87. DOI: https://doi.org/10.1590/S0102-311X2002000500031.

4. Riggs P. Non-medical use and abuse of commonly prescribed medications. Curr Med Res Opin. 2008;24(3):869-77.

DOI:

https://doi.org/10.1185/030079908X273435

5. Catlin DH. Anabolic androgenic steroids. In Karch SB. Drug abuse handbook. Boca Raton: CRC Press; 1998.

6. Karbalay D, Noorafshana. Stereological study of the effects of nandrolone decanoate on the rat protaste. Micron. 2006;37:617-23. DOI: https://doi.org/10.1016/i.micron.2006.02.005

7. Kicman AT. Pharmacology of anabolic steroids: $\mathrm{Br} \mathrm{J}$ Pharmacol. 2008;154(3):502-21. DOI: https://doi.org/10.1038/bjp.2008.165

8. Camargo ICC, Gaspar ALC, Frei F, Mesquita SFP. Efeitos dos esteroides anabólicos androgênicos sobre 
o útero e parâmetros reprodutivos de ratas adultas. Rev Bras Ginecol Obst. 2009;31(9):453-60. DOI: https://doi.org/10.1590/S0100-72032009000900006

9. Silva PRP, Danielski R, Czepielewski MA. Esteroides anabolizantes no esporte. Rev Bras Med Esporte. 2002;8(6):235-43. DOI: https://doi.org/10.1590/S1517-86922002000600005

10. Araujo LR, Andreolo J, Silva MS. Utilização de suplemento alimentar e anabolizantes por praticantes de musculação nas academias de Goiânia-GO. Rev Bras Ciênc Mov. 2002;10:13-8.

11. Iriart JAB, Chaves JC, Orleans RG. Culto ao corpo e uso de anabolizantes entre praticantes de musculação. Cad Saúde Pública. 2009;25(4):773-82. DOI: https://doi.org/10.1590/S0102-311X2009000400008

12. Lise MLZ, Silva TSDG, Ferigolo M, Barros HMT. O abuso de esteroides anabólico androgênicos em atletismo. Rev Assoc Méd Bras. 1999;45(4):364-70. DOI: $\quad$ https://doi.org/10.1590/S0104$\underline{42301999000400014}$

13. Silva $A O$, Yonamine $M$, Oga $S$. Dopagem no esporte. In. Silva AO. Fundamentos de toxicologia. São Paulo: Atheneu; 2003.

14. Bahrke MS, Yesalis CE. Abuse of anabolic steroids and related substances in sport and exercise. Curr Opin Pharmacol. 2004;4:614-20. DOI: https://doi.org/10.1016/i.coph.2004.05.006

15. Ferreira LO, Couto BP, Szmuchrowski LA, Drummond MDM. Efeitos colaterais associados ao uso de esteroides anabolizantes andrógenos auto relatados por praticantes de musculação do sexo masculino. Rev Bras Ciên Saúde. 2014;18:35-42. DOI: https://doi.org/10.4034/RBCS.2014.18.S4.05

16. Silva LSMF e Moreau RLM. Uso de esteroides anabólicos androgênicos por praticantes de musculação de grandes academias da cidade de São Paulo. Rev Bras Ciên Farmac. 2003;39(3):327-33. DOI: https://doi.org/10.1590/S1516-93322003000300012

17. Carneiro JMA, Silva AC, Almeida MM. Nível de conhecimento e ocorrência do uso de anabolizantes entre praticantes de musculação. Rev Cient FAGOCSaúde. 2016;1(1):37-40.

18. Mineiro L, Silva JC, Silva JOM, Rocha RE. Uso de esteroides por frequentadores de academias de musculação na cidade de Caçador-SC. Rev Bras Prescr Fisiol Exerc. 2016;9(53):321-7.
19. Frizon F, Macedo S, Yonamine $M$. Uso de esteróides andrógenos anabólicos por praticantes de atividade física das principais academias de Erechim e Passo Fundo-RS. Rev Ciên Farmac Bás Apl. 2009;26(3):227-32.

20. Santos AF, Mendonça PMH, Santos LDA, Silva NF, Tavares JKL. Anabolizantes: conceitos segundo praticantes de musculação em Aracaju-SE. Psicol Estudo. 2006;11(2):371-80. DOI: https://doi.org/10.1590/S1413-73722006000200016

21. Moraes MP. Aspectos Motivacionais relacionados ao uso de esteroides Anabolizantes. [Monografia]. Escola de Educação Física, Universidade Federal do Rio Grande do Sul, Porto Alegre, 2014.

Recebido para publicação em 04/08/2017

Revisado em 14/08/2017

Aceito em 17/08/2017 